\title{
Static Power Minimization in Current-Mode Circuits
}

\author{
M. S. Bhat and H. S. Jamadagni \\ Centre for Electronics Design and Technology, \\ Indian Institute of Science, \\ Bangatore-560012, India \\ \{msbhat,hsjam\}@cedt.iisc.ernet.in
}

\begin{abstract}
We propose a method involving selective signal gating to minimize power dissipation in current-mode CMOS analog and multiple-valued logic (MVL) circuits employing a stack of current comparators. First, we present an approximation model for current in a current comparator circuit. Power reduction is achieved by turning off the redundant comparator circuits using a switch-architecture. Simulations are carried-out for current-mode flash ADC designs and literal generating circuits for MVL to validate the method.
\end{abstract}

\section{INTRODUCTION}

Wide spread interest in wireless communication and portable computing has created a critical need for low-power low-voltage analog and digital integrated circuits. The three components of power dissipation in CMOS logic are switching, static (or leakage) and short-circuit. Of the three components, the dominant component in analog and multiple-valued logic circuits being static power dissipation. Therefore, static power reduction is an important optimization goal in analog and MVL circuit design.

Current-mode circuit techniques, which process the active signals in the current domain, offer a number of advantages [1]. Current comparator is a fundamental component of current-mode analog integrated circuits. A critical design aspect for comparator is good trade-off between sensitivity, speed and power dissipation. In the last decade, several comparator architectures have focused to address some of these issues [2]-[5]. When eircuits employ a set of comparators for purposes such as those in flash ADC and literal based multiple-valued logic modules, the comparator set generates a thermometer code that reflects the input signal amplitude. As the input signal amplitude increases, more and more comparators are turned on thereby establishing a large static current from power supply to ground. Since we are interested in the outputs of only those comparators whose output changes from zero to one for the given input, we can, in principle, turn-off many of the comparators whose outputs are already high and that they do not contribute any information to the final digital value. This can be accomplished by introducing switches at appropriate places to turn off the current drawn by such comparators without affecting the final output value.

\section{POWER Dissipation IN CURRENT COMParators}

Power dissipation in CMOS digital circuits is given by the well-known expression,

$$
P=C \cdot V_{D D}^{2} \cdot f \cdot \alpha+t_{s c} \cdot I_{p e r t i} \cdot V_{D D} \cdot f \cdot \alpha+I_{\text {leatage }} \cdot V_{D D}
$$

The three terms on the right hand side of (1) correspond to dynamic power, short-circuit power and leakage power respectively. $C, f, \alpha$ and $t_{s c}$ are the total switching capacitance, clock frequency, switching activity factor and the time during which a short circuit exists between supply and ground respectively.

In the case of analog circuits, the static power dissipation due to finite resistance path from supply to ground (resulting from triode and saturation region operation of the transistors) dominates and hence, as a first approximation, we have neglected the dynamic, short circuit and leakage power components in this work.

Fig. 1 shows one of the simplest current comparator circuits, proposed by [2]. It consists of current mirrors to replicate the input current signals and compares the current signal $I_{l}$ with a predetermined reference $I_{r l}$ and generates a two-level voltage signal $V_{\text {mut }}$. The comparator circuit comprises just two transistors, $M_{N O}$ and $M_{P 0}$. Assuming $M_{N O}$ to be identical to $M_{R}, M_{P O}$ to $M_{\sigma}$, and that there are no errors due to lithographic effects in forming the transistors, the static currents in the comparator for the three modes of operation are listed below.

Case 1. $I_{i}=I_{r l}$. Both $M_{N O}$ and $M_{F 0}$ are in saturation mode and $V_{\text {our }} \approx V_{D D} / 2$.

Case 2. $I_{I}>I_{r i}$. $\quad M_{N O}$ is in the triode mode, $M_{P O}$ is in saturation mode, and $V_{\text {out }}$ is set at its low level.

Case 3. $I_{l}<I_{r i} . \quad M_{N O}$ is in the saturation mode, $M_{P O}$ is in triode mode, and $V_{o u t}$ is set at its high level.

The circuit path from supply to ground in the comparator branch will carry a current equal to $I_{\text {ref }}$ in case 1 and almost equal to $I_{r e f}$ in case 2 and equal to $I_{i n}$ in case 3 .

In case 1, the current in the comparator branch is given by

$$
I_{r 1}=\frac{\mu_{P} C_{o f p}}{2} \frac{W_{P}}{L_{P}}\left(V_{G S P}-V_{T P}\right)^{2}=\frac{\mu_{N} C_{a x p}}{2} \frac{W_{N}}{L_{N}}\left(V_{G S N}-V_{T N}\right)^{2}
$$

where $\mu, C_{o x}, W, L, V_{u s}$ and $V_{I}$ are the mobility, gate capacitance, channel width, channel length, gate to source voltage and threshold voltage respectively for the PMOS and the NMOS transistors.

In case 2 (case 3), the voltage drop across $M_{P O}\left(M_{N O}\right)$ is much larger than the drop across $M_{a}\left(M_{R}\right)$ and results in channel length modulation [6]. This introduces errors in current mirroring. As a second approximation, we neglect this error and assume a relation between $I_{l}$ and $I_{i n}$ as depicted in fig. 2.

Section IIl gives a description of the switch architecture used for power optimization. 


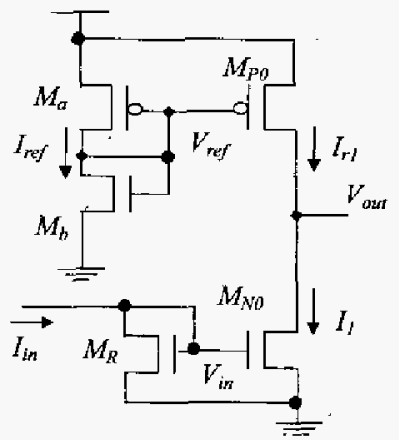

Fig. 1. Simple current comparator with a reference current generator

\section{SWITCH ARCHITECTURE}

Our power optimization scheme assumes a circuit topology wherein a number of current comparators are used to compare the input current signal to generate a thermometer code. Fig. 3(a) shows the schematic of power optimization scheme using the switch and $3(\mathrm{~b})$ shows the circuit diagram of the switch. In fig. 3(a), each comparator (CMP) is equal to the $M_{P O}$ and $M_{N O}$ connection of fig. 1 with the gates of PMOS and NMOS transistors connected to $V_{\text {ref }}$ and $V_{i n}$ respectively. For a given input signal, let us assume that the comparator outputs from $\mathrm{I}$ to $\mathrm{J}$ are low, and above $\mathrm{J}$ are high (thermometer code). In this case, $\mathrm{J}^{\text {th }}$ output is of significance and all lower outputs do not carry any information. In this scenario, we can turn off the PMOS devices of lower comparators by switching their gates to $V_{\text {ud. }}$. This, however, will not alter the comparator outputs, but stop the current drawn from the supply and thereby reducing the power dissipation.

\section{Current-Mode Circuit Examples}

In this section, the technique suggested in section III is applied for power optimization in two example circuits, viz., current-mode flash ADC circuit and window-literal eircuit used in MVL function realization.

\section{A. Flash $A D C$}

We designed a 7-bit current-mode flash ADC using current comparators. The ADC was designed for a dynamic range

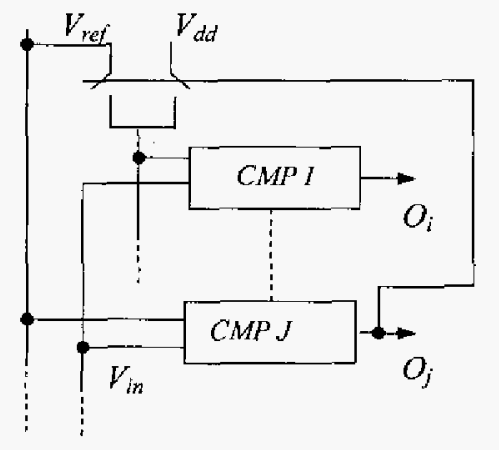

Fig. 3(a). Power optimization scheme

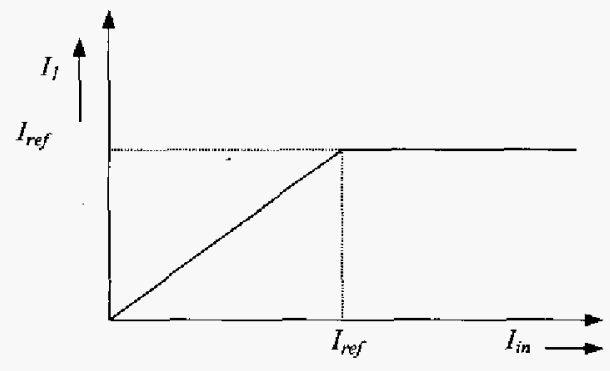

Fig. 2. Relation between $I_{i n}$ and $I_{I}$

of $32 \mu \mathrm{A}$. The circuit schematic of the basic ADC block generating the thermometer code is shown in figure 4 . The reference current block (block $\mathrm{A}$ ) generates a reference current $I_{r e f}$ and the input block (block B) receives the input current $I_{\text {input }}$. The current comparator block consists of 128 current comparators comprising 128 PMOS and NMOS current mirrors. The reference current, $l_{r \varepsilon f}$ is replicated by the PMOS current mirrors such that the current flowing in these mirrors progressively increase from $I_{0}$ (first mirror) to $I_{2,7}$ (last mirror). The input current, $I_{\text {input }}$, is also replicated by NMOS current mirrors to generate 128 identical copies. These are then compared with the reference currents to generate the thermometer code. Gain boosters (inverters) are used to shape this output binary signal.

The ADC is designed using 0.7- $\mu \mathrm{m}$ MIETEC CMOS technology. We could achieve a maximum sampling speed of $80 \mathrm{Ms} / \mathrm{sec}$ at a power consumption of $78 \mathrm{~mW}$.

To optimize power, we-modified the ADC architecture by employing power saving switch as shown in fig. 5 . The circuit differs from the one depicted in fig. 4 in that it includes switches to turn-off the redundant currents being drawn from $V_{d d}$. 128 comparators of the ADC are grouped in such a way that each group consists of, say, $k$ comparators. Switches can be introduced in all groups except the last group. For a given value of input current, full reference current gets established in those comparators whose outputs have gone high, whereas, only a portion of the reference currents flows in the remaining (higher order) comparators, and hence their output is at logic low (refer fig. 4). Thus, if the outputs of $\mathrm{j}^{\text {th }}$ group of comparators are high, then all the lower group outputs also will be high. In this scenario, we can turn-off reference PMOS transistors of lower group of comparators by switching their gates to $V_{d d}$ using the outputs of $j^{\text {th }}$ group of comparators, there by forcing the reference currents in these

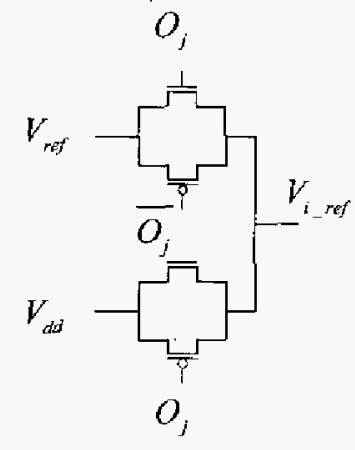

Fig. 3(b). Circuit diagram of the switch 


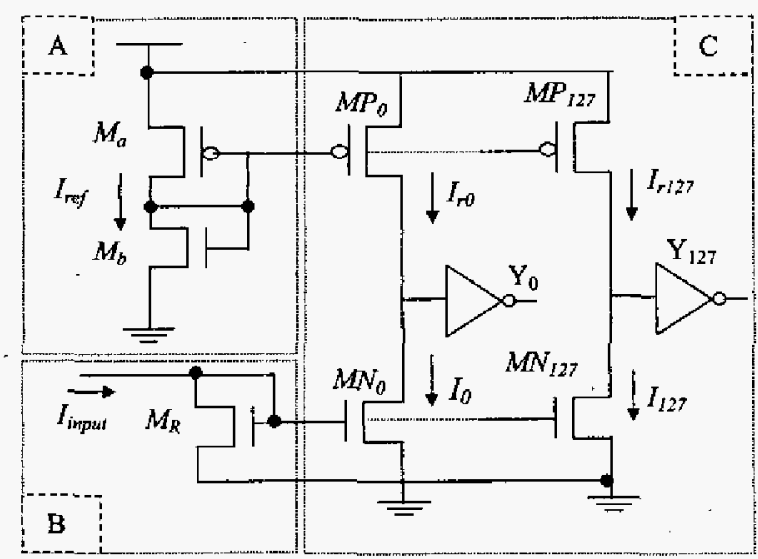

Fig. 4. Circuit schematic of basic ADC block

transistors to go zero without altering the output logic value.

Power saving in ADC comes at the cost of increased delay. It is found that the number of comparators per group have a bearing on both power saving and delay. Fig. 6 shows power delay trade-off as a function of number of comparator groups.

Simulation results show that the average power can be reduced by $23 \%$ with a delay penalty of around $9 \%$, which corresponds to 5 comparator groups (each group consisting of around 26 comparators). Simulation results showing total current drawn by the set of comparators when the input signal is swept from zero to $32 \mu \mathrm{A}$ is depicted in fig. 7 . The numbers on the right side of the graph indicate the number of comparators in each group.

\section{B. Literal generating Circuit for Multiple-Valued Logic}

Multiple-Valued Logic designs have been receiving considerable attention over a couple of decades. The signal processing on the basis of the multiple-valued logic is carried out using multiples of logic levels and thresholds, in contrast to binary logic with its two states. Most of the designs are current-mode circuits because of their advantages over voltage-mode circuits [7]-[8].

When literals are used to realize MVL functions as in the case of self-restoring logic architecture [9], it is necessary to generate or detect the logic values of variables. Current signals are used to represent the logic levels with assignments such as Logic $0=0$ (no current), Logic $1=I_{0}$, Logic $2=2 I_{0}$,

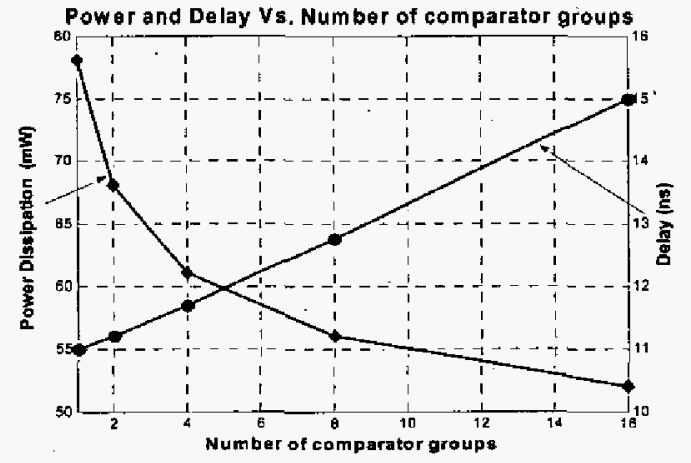

Fig. 6. Power-Delay Trade-off

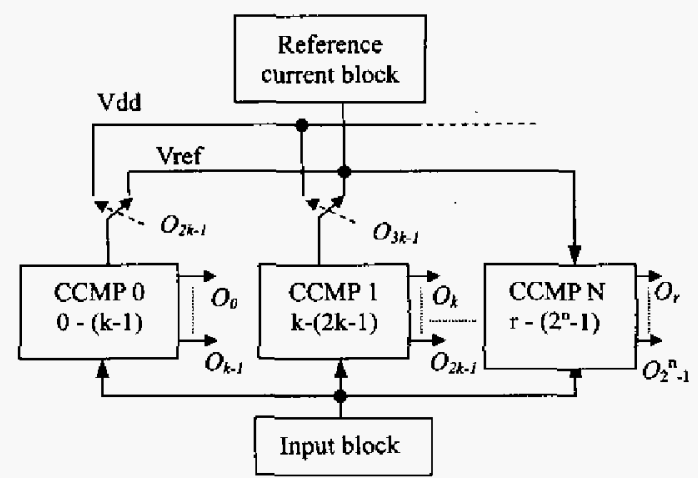

Fig. 5. Power optimized ADC schematic

and so on with $I_{0}$ equal to, say, $10 \mu \mathrm{A}$. An obvious choice to determine the current level is a current comparator circuit.

Except for a few additional binary logic gates, the literal generating circuit of an $m$-valued logic is very similar to that shown in fig. 4. However, the total number of current comparators would be far too less than that used in an ADC. Therefore, we can introduce switches to turn off every comparator except the last one, for power optimization. For an $m$-valued, $n$-variable logic function, the total number of comparators would at most be equal to $(m-I) n$.

Power dissipated in the comparator block depends on the literal values present in the function. Maximum current drawn by the input block per variable is given by,

$$
I_{\text {Max }}=\sum_{k=1}^{m-k}\left[\frac{k(k+1)}{2}\right] I_{0}
$$

where $I_{0}$ is the reference current.

For functions with each variable assuming equi-probable literal values, the average current drawn per variable is given by,

$$
I_{\Delta V \hat{S}}=\frac{m+1}{2} I_{\text {in }}+\frac{1}{2}\left(\frac{(m+1)(m+2)}{6}+1\right) I_{0}
$$

For a function with $n$-variables,

$$
I_{\text {Tor }}=n \cdot I_{\text {AVG }}
$$

Then the average power dissipated by the comparator blocks is,

$$
P_{C M}=I_{A K G} V_{D D}
$$

When switches are introduced for each variable to turn off the redundant comparators as discussed in the ADC case, the

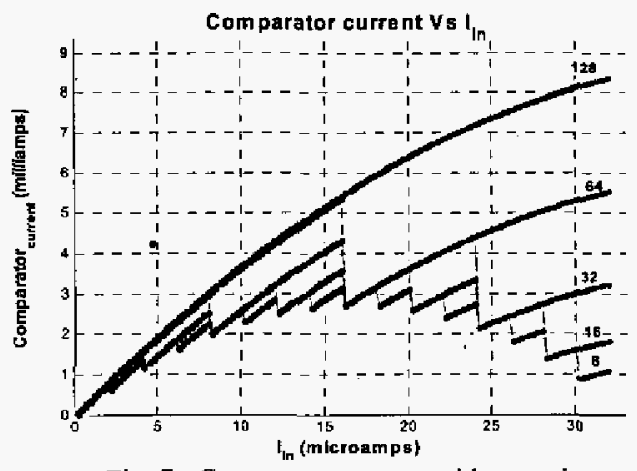

Fig. 7 Comparator current with grouping 
average current drawn by the comparator block for a variable taking an equi-probable value becomes,

$$
\overline{I_{A V G}}=\frac{m+1}{2}\left[I_{i m}+I_{0}\right]
$$

Comparing equation (4) and (7), it is seen that the static current can be reduced significantly in functions with radix 4 and above. The actual reduction in power depends on the probability of the value that each variable takes in a given function.

We performed simulation experiments on a number of randomly generated 2-variable, 4 - and 5-valued functions implemented on $0.13 \mu \mathrm{m}$ CMOS process in self-restoring logic style. All circuits were excited with the same input signals with equal literal probabilities. Some example functions are listed in table 1. Theoretically, a saving of around $20 \%$ can be achieved for these functions.

Fig. 8(a) and (b) are the simulation results showing the currents flowing in the three comparator circuits without and with the power optimization switch, respectively. Note that the switches are employed only in the first and the second comparators and are activated by the outputs of the second and the third comparators respectively. A power reduction in the range of $5 \%-19 \%$ was observed in these experiments. The low value of power reduction was attributed to functions with variables not taking all logic values. Static power saving in CMOS MVL circuits comes at the cost of increased delay of around $6 \%$.

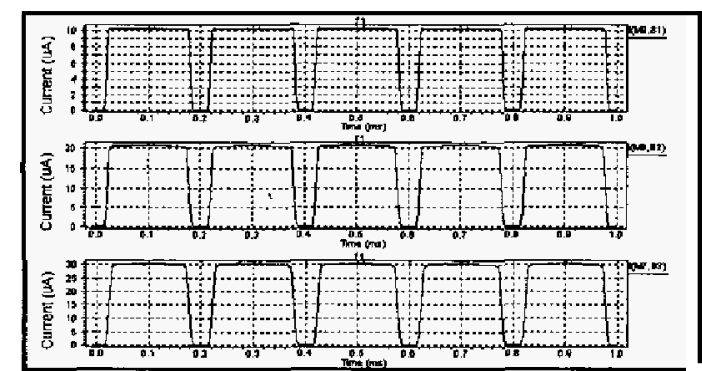

Fig. 8(a). Simulationresults of MVL function $f l$ without the power optimizing switch

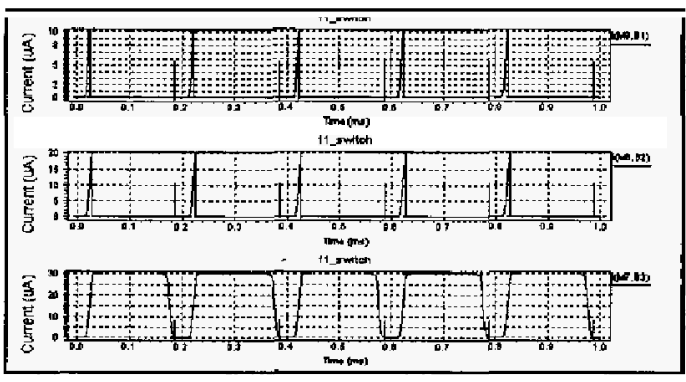

Fig. 8(b). Simulation results of MVL function $f 7$ with the power optimizing switch

Current cornparator circuits are also employed in many other analog and fuzzy logic function realizations and there is scope for power reduction using the method suggested in this work. However, some problems may arise in precision analog and digital circuits due to charge injection with the insertion of the switches.

\section{CONCLUSIONS}

Power optimization scheme, using switch architecture, in analog and multiple-valued logic circuits employing a series of current comparators is presented. A 7-Bit Current-mode Flash ADC and a number of 2-variable 4- and 5-valued functions were designed and simulated with and without power optimization scheme. Simulation results show that static power dissipation by the comparators can be significantly reduced with a nominal delay penalty.

In the case of $A D C$, power delay trade-off can be achieved to give best results by varying the number of comparators in each group. A Power saving of $23 \%$ was achieved with an additional delay of $9 \%$ in flash ADC, while 5\%-19\% power saving was reported with an additional delay of $6 \%$ in the case of multiple-valued functions.

Table 1. Some 2-VARIABLE4-Valued EXAmple Functions Used IN THE EXPERIMENT

\begin{tabular}{|c|c|c|c|}
\hline \multirow{2}{*}{ Function } & \multicolumn{3}{|c|}{ Power Dissipation $(\mu \mathrm{W})$} \\
\cline { 2 - 4 } & Unoptimized & Optimized & $\%$ Saving \\
\hline$f_{1}$ & 210 & 181 & 13.8 \\
\hline$f_{2}$ & 214.9 & 192.3 & 10.5 \\
\hline$f$ & 212 & $I 78.5$ & 15.8 \\
\hline$g$ & 208.6 & 171.2 & 17.93 \\
\hline
\end{tabular}

\section{REFERENCES}

[1] C. Toumazou, F. J. Lidgey, and D. G. Haigh, ed., Analogue IC design: the current-mode approach, IEE circuits and systems series 2, 1990.

[2] Freitas, D. A. and Current, K. W., "CMOS current comparator circuit", Electronic letters 19(17), pp. 695-697, 1983

[3] Taff, H., "Novel approach to high speed CMOS-current comparators", Electronic letters 28(3), pp. 3 10-312, 1992

[4] Tang, A. T. K. and Toumazou, C., "High performance CMOS current comparator", Electronic letters 30(I), pp. 5-6,1994

[5] Chen, L., Shi, B., and Lu, C., "Circuit design ofa high speed and low power CMOS continuous-time current comparator", Analog Integrated Circuits and Signal Processing, Kluwer Academic Publishers, 2001, pp. 293-297.

[6] Gray, P. R., Hurst, P. J., Lewis S. H. and Meyer, R. G. "Analysis and Design of Analog Integrated circuits", $4^{\text {th }}$ Ed., John Wiley and Sons Inc., 2001.

[7] K.W. Current, "Current-mode CMOS multiple-valued logic circuits", IEEE J. Solid State Circuits, vol. 29, no. 2, pp. 95-107, Feb.1994

[8] A.K.Jain et al., "CMOS Multiple-valued logic design - Part I: Circuit implementation", IEEE Trans. On Circuits and Systems - I: Fundamental theory ond applications, vol. 40, no. 8, pp. 503-514, Aug. 1993.

[9] Teng, D. H. Y., and Bolton, R. J., "A self-restored Current-mode CMOS Multiple-Valued Logic design Architecture", Proceedings of the $7^{\text {th }}$ IEEE Pacific Rim Conference on Communications, Computers and Signal processing, (Victoria, Canada), pp. 436-439, Aug. 22-24, 1999. 